\title{
Screening of significant oncogenic changes in air pollution-related lung cancer in Chinese population
}

\author{
Madiha Kanwal ${ }^{1,2}$, Xiaojie Ding ${ }^{1}$, Weiming Lin $^{3}$, Yi Cao ${ }^{1,3}$ \\ ${ }^{1}$ Laboratory of Molecular and Experimental Pathology, Kunming Institute of Zoology, Chinese Academy of Sciences, Kunming, China; ${ }^{2}$ Kunming \\ College of Life Sciences, University of Chinese Academy of Sciences, Kunming, China; ${ }^{3}$ Department of Veterinary Medicine, College of Life \\ Sciences, Longyan University, Longyan, China \\ Contributions: (I) Conception and design: Y Cao, M Kanwal; (II) Administrative support: Y Cao, W Lin; (III) Provision of study materials or patients: \\ Y Cao, W Lin; (IV) Collection and assembly of data: M Kanwal, X Ding, Y Cao; (V) Data analysis and interpretation: M Kanwal, X Ding, Y Cao; (VI) \\ Manuscript writing: All authors; (VII) Final approval of manuscript: All authors. \\ Correspondence to: Prof. Dr. Yi Cao. Laboratory of Molecular and Experimental Pathology, Kunming Institute of Zoology, Chinese Academy of \\ Sciences, 32 Jiaochang Donglu, Kunming, China. Email: yicao1212@yahoo.de.
}

Background: Air pollution-related lung cancer has been considered as a deteriorating public health problem worldwide, particularly in developing countries. The highly air polluted regions in China particularly Xuanwei and Fuyuan counties have markedly high lung cancer rates and are considered as good models to study air pollution-related lung cancer. The present study investigated the clinically significant oncogenes in air pollution-related lung cancers.

Methods: A combination of reverse transcriptase PCR (RT-PCR) and DNA sequencing was applied to examine the expression, mutations, or fusions of target genes, including human epidermal growth factor receptor 2 (HER2), echinoderm microtubule-associated protein-like 4-anaplastic lymphoma kinase (EML4$A L K)$, and cluster of differentiation 74-ROS proto-oncogene 1 (CD74-ROS1).

Results: Of the 82 patients with lung cancer, 20.7\% (17/82) exhibited HER2 up-regulation, and 1.2\% (1/82) harbored HER2 insertion at exon 20. HER2 overexpression was not associated with air pollution levels and smoking status. A total of 6.1\% (5/82) samples exhibited ALK gene rearrangements; two belonged to EML4-E2 + ALK-E20 and three were EML4-E13 + ALK-E20; all the five cases occurred either in smokers or in patients from the most air-polluted region. A total of 3.6\% (3/82) carried the CD74-ROS1 fusion gene (CD74-E6 + ROS1-E34); the fusion event was not statistically associated with air pollution levels.

Conclusions: The high rates of prevalence indicated in the present study suggest that the screening of HER2 overexpression and EML4-ALK fusion events may assist in guiding treatment in air pollution-related lung cancer; the RT-PCR-based test proposed in this study may be a useful tool in clinical applications to screen these genetic changes.

Keywords: Air pollution; lung cancer; reverse transcriptase PCR (RT-PCR); HER2; EML4-ALK; CD74-ROS1

Submitted Jul 14, 2019. Accepted for publication Jan 03, 2020.

doi: $10.21037 /$ tcr-19-1314

View this article at: http://dx.doi.org/10.21037/tcr-19-1314

\section{Introduction}

Lung cancer is one of the most common malignancies and has been the leading cause of cancer-associated mortality worldwide. An estimated 1.8 million incident lung cancer cases occurred in 2012 , accounting for $\sim 13 \%$ of total cancer diagnoses (1). As indicated by the Global Burden of Disease study 2020, the health care burden and costs related to lung cancer are significant on a global scale (2). Lung cancer is categorized into two primary histological types: small cell lung cancer (SCLC) and non-small cell 
lung cancer (NSCLC). NSCLC is additionally divided into adenocarcinoma (AD), squamous cell carcinoma (SCC) and large cell carcinoma (LCC). Approximately $85 \%$ of lung cancer cases are NSCLC and SCLC accounts for approximately $15 \%$ of cases (3). The major etiological factors of lung cancer are smoking and air pollution. Although smoking is one of the most important causes of lung cancer, however $25 \%$ of lung cancer cases are observed in never-smokers worldwide in 2013 (4). If lung cancer in non-smokers is considered as a separate disease, this malignancy ranks seventh among the fatal types of cancer worldwide (5). Air pollution induces more than 200,000 lung cancer deaths globally (6). The International Agency for Research on Cancer (IARC), World Health Organization, has classified air pollution as Group 1 carcinogenic agent for humans $(7,8)$. In addition, industrial developments have increased air pollutant concentrations in certain regions, cities, and countries, particularly in developing countries (8). For example, air pollution has become the fourth greatest threat to the health in the Chinese population; 350,000-500,000 Chinese people succumb prematurely each year due to air pollution (9). According to the latest statistics on cancer in China in 2017, lung cancer has become the malignant tumor with the highest morbidity and mortality rates in China, and these high progression rate is more severe due to increasingly high levels of air pollution compared with previous cases in developing countries and in other regions $(10,11)$. However, the molecular characteristics of air pollution-related lung cancer remain poorly understood. Xuanwei City and Fuyuan County in Yunnan Province in China are heavily air-polluted regions, with particularly high lung cancer rates among smokers and non-smokers; as such, these regions are considered good models to investigate air pollutionrelated lung cancer (11-13). In Xuanwei and Fuyuan, the lung cancer incidence is 4-5 times higher compared to the national average; with high prevalence rate in non-smoking females. The high risk is attributed to exposure to indoor and outdoor air pollution caused by smoky coal burning. In smoky coal burning, high concentrations of carcinogenic substances, including fine particulate matter $(\mathrm{PM} ; \leq 2.5 \mu \mathrm{m}$ in diameter, PM2.5) and coarse PM $(\leq 10 \mu \mathrm{m}$ in diameter, $\mathrm{PM} 10)$, and polycyclic aromatic hydrocarbons (PAHs), are released $(13,14)$. The data from Xuanwei have been cited in the IARC monograph (6); and indoor emissions from household coal combustion are classified as carcinogenic to human health (Group 1).

The accumulation of genetic alterations or specifically hazardous mutations in vital genes, which control critical cellular processes, including growth, proliferation, differentiation, and survival, is considered as a major cause of oncogenesis (15). Certain harmful genetic changes may be inherited as hereditary factors from parents, while most of the changes may manifested as somatic mutations, frequently and predominantly caused by random errors during DNA replication and environmental carcinogens (16). Therefore, these genetic changes should be investigated to understand carcinogenesis, and to diagnose, treat, and prevent cancers. The number of identified oncogene drivers of lung cancer has increased in previous decades; these oncogene drivers include epidermal growth factor receptor $(E G F R)$, tumor protein 53 (TP53), v-Raf murine sarcoma viral oncogene homolog B $(B R A F)$, v-Ki-Ras2 Kirsten rat sarcoma viral oncogene homolog $(K R A S)$, and human epidermal growth factor receptor 2 (HER2), as well as anaplastic lymphoma kinase $(A L K)$ and ROS protooncogene 1 (ROS1) gene rearrangements (17). Certain oncogenes function as good therapeutic targets, in addition to oncogenic drivers in carcinogenesis. Patients with specific genetic alterations exhibit increased sensitivity to targeted therapy than to other therapeutic strategies $(18,19)$. For example, patients are responsive to EGFRtargeted therapies, including erlotinib and gefitinib treatments which results in marked antitumor activity in patients due to EGFR overexpression (20); patients are sensitive to Herceptin (trastuzumab) treatment due to HER2 overexpression (18); similarly, patients are responsive to crizotinib treatment and exhibit significantly improved progression free survival rates due to mutations leading to gene fusions in the echinoderm microtubule associated protein-like 4 (EML4)-ALK(19).

Comprehensive profiles of certain genes including EGFR, K-RAS and TP53 have been studied in air pollution-related lung cancer in Xuanwei and Fuyuan $(16,20,21)$; therefore, the present study excluded these genes from additional screening. In the present study, lung cancer samples were obtained from Chinese populations in Xuanwei and Fuyuan to investigate the molecular features of air pollution-related lung cancer. HER2, BRAF, and EML4-ALK and cluster of differentiation 74-ROS1 (CD74-ROS1) fusion genes, were selected from a group of known oncogene drivers, as they are clinically important oncogenes, and molecular-targeted medicines are available for therapies. At present, numerous techniques have been developed to enhance the screening of these oncogenes. For instance, fluorescence in situ hybridization (FISH) and 
immunohistochemistry (IHC) are conventional methods to detect these genetic changes, however these methods exhibit limitations in clinical practice $(22,23)$. Therefore, more effective and economical screening tools are necessary to easily examine these oncogenes. Polymerase chain reaction (PCR)-based tests provide several advantages compared to FISH and IHC; for example, large number of samples can be processed in a single round of testing, these techniques can be easily performed and few facilities are required; PCR-based tests are also rapid and economical (24-26). Thus, in the present study, a combination of reverse transcriptase PCR (RT-PCR) and DNA sequencing was applied to examine the expression, mutations, and fusions of target genes. This study primarily aims to define the status of clinically significant oncogenes in air pollution-related lung cancers, and to establish a simple and sensitive test to screen patients with particular genetic changes.

\section{Methods}

\section{Cell culture}

A total of 13 cell lines, comprising 11 cancerous and two immortal human bronchial epithelial cell lines (16HBE and Beas-2B), were used in the present study. The 11 lung cancer cell lines included AD (SPC-A-1, GLC-82, A549, XLA-07, XL-JT), SCC (EPLC-32M1), LCC (801D, NCI-H460, 95-D), mucoepidermoid lung carcinoma (NCI-H292), and SCLC (NCI-H446). The Xuanwei lung cancer cell line (XL-JT) was identified using short tandem repeat typing (27). All 11 cancer cell lines were cultured in RPMI-1640 (GE Healthcare Life Sciences, Logan, UT, USA) and the two immortal human bronchial epithelial cell lines were cultured in Dulbecco's modified Eagle's medium (GE Healthcare Life Sciences) with 10\% fetal bovine serum (Thermo Fisher Scientific, Waltham, MA, USA) and incubated at $37{ }^{\circ} \mathrm{C}$ in a humidified atmosphere of $5 \% \mathrm{CO}_{2}$.

\section{Clinical samples}

Primary lung tumor and adjacent non-malignant tissue samples were collected from the Yunnan First People's Hospital and the Yunnan Tumor Hospital, Kunming from March 2006 to January 2015; all the samples were obtained from the patients who provided written informed consent. None of the patients had undergone chemotherapy or radiotherapy prior to surgery. The tissue samples were stored in liquid nitrogen at the time of resection. A second pathological diagnosis additionally confirmed the histological characteristics of the tumors in accordance with the WHO classification (3). The hematoxylin-eosinstained sections were observed through light microscopy to evaluate the cancer cell ratio and the necrosis rate. All tumor samples that comprised $>70 \%$ cancer cells and exhibited $<10 \%$ necrosis were included in the study. All of the selected patients resided in Xuanwei City and Fuyuan County in Yunnan Province, China. This whole geographical area was divided into three regions: A (heavily polluted; $>170 \mathrm{ng} / \mathrm{m}^{3}$ ), B (moderate; $60-170 \mathrm{ng} / \mathrm{m}^{3}$ ), and C (less polluted; $<60 \mathrm{ng} / \mathrm{m}^{3}$ ), according to concentration of indoor and outdoor benzo(a)pyrene (Bap; the best index of PAHs carcinogenicity) $(14,16,27,28)$. The age of the patients ranged from 34-76 years, with median age of 55 years. As being a smoker or living in highly polluted area may increase exposure of the respiratory system to high level of air carcinogens, subjective behavior (smoking) and objective factors (air pollution) were combined to determine total exposure degree in the present study. The groups of high total exposure included patients living in more polluted region, regions $\mathrm{A}$ and $\mathrm{B}$, and/or smokers, while the group of low total exposure included patients reside in the relatively clean region $\mathrm{C}$ and non-smokers. This study was approved by the Ethics Committee for Human Medicine Research at the Kunming Institute of Zoology, Chinese Academy of Sciences (Permit Number: SYDW-2012010). All patient records and information were anonymized and de-identified prior to analysis. Research was conducted in accordance with the principles of the Declaration of Helsinki (as revised in 2013).

\section{Reverse transcriptase PCR (RT-PCR)}

Total RNA was extracted from the tissues and the cell lines by using TRIzol reagent (Invitrogen, Carlsbad, CA, USA) and concentration was measured at 260 and $280 \mathrm{~nm}$ using a spectrophotometer (Bio-Rad Laboratories, Hercules, CA, USA); then, $2 \mu \mathrm{g}$ total RNA was reverse-transcribed into a single-stranded cDNA by using a reverse transcription kit (Promega Corporation, Madison, WI, USA). A total of $1 \mu \mathrm{g}$ cDNA was used as a template, and RT-PCR was then performed to examine the expression of $H E R 2$ and the prevalence of EML4-ALK and CD74-ROS1 fusion genes. The details of all the oligonucleotide primers for PCR and sequencing are summarized in Table 1. PCR primers were also used for sequencing; however, a separate set of 
Table 1 Primer information for RT-PCR in this study

\begin{tabular}{ll}
\hline Targets & Primer sequence $\left(5^{\prime} \rightarrow 3^{\prime}\right)$ \\
\hline HER2 & F: CCCTCTGACGTCCATCATCT \\
& *F: GGTCTTTGGGATCCTCATCA \\
& R: GCAGGGTCTGGACAGAAGAA \\
BRAF & F: TCAGAAGACAGGAATCGAATGA \\
& R: TCATACAGAACAATTCCAAATGC \\
& *R: GATGACTTCTGGTGCCATCC \\
EML4-E2-ALK-E20 & F: TCACTGTGCTAAAGGCGGCTTTGG \\
$(29)$ & R: CAGGGCTTCCATGAGGAAATCCAG \\
EML4-E13-ALK-E20 & F: CCACACCTGGGAAAGGACCTAAAG \\
$(29)$ & R: CAGGGCTTCCATGAGGAAATCCA \\
CD74- ROS1 & F: CCTGAGACACCTTAAGAACACCA \\
& R: TGAAACTTGTTTCTGGTATCCAA \\
GAPDH & F: TGTTGCCATCAATGACCCCTT \\
& R: CTCCACGACGTACTCAGCG
\end{tabular}

*, used for DNA sequencing.

sequencing primer were used for the genotyping of HER2 and $B R A F$ genes. PCR amplification was performed in a Bio-Rad thermocycler (Bio-Rad Laboratories) with 1 cycle of denaturation at $95^{\circ} \mathrm{C}$ for $5 \mathrm{~min}$, followed by 35 cycles of $95{ }^{\circ} \mathrm{C}$ for $1 \mathrm{~min}$, annealing at $55-60^{\circ} \mathrm{C}$ for $30 \mathrm{sec}$ and extension at $72{ }^{\circ} \mathrm{C}$ for $1 \mathrm{~min}$. Final extension was performed at $72{ }^{\circ} \mathrm{C}$ for $10 \mathrm{~min}$. The RT-PCR products were analyzed through $1 \%$ agarose gel electrophoresis and stained with ethidium bromide. GAPDH was used as an internal control. Positive results were repeated in triplicate to confirm the results.

HER2, BRAF, and EML4-ALK were amplified in a $25 \mu \mathrm{L}$ reaction mixture using a Taq Polymerase from Thermus aquaticus kit (Sigma-Aldrich, Darmstadt, Germany); the mixture contained $0.5 \mu \mathrm{L}$ each of forward and reverse primers $(10 \mu \mathrm{M}), 1.5 \mu \mathrm{L}$ cDNA template and $\mathrm{H}_{2} \mathrm{O}$ was added to obtain the final reaction volume. In addition, $2 \mu \mathrm{L}$ of $5 \mathrm{M}$ betaine (Sigma-Aldrich) was added to facilitate an enhanced amplification of EML4-ALK. To facilitate an enhanced amplification of CD74-ROS1, a Taq PCR kit (cat. No., E5000S; New England Biolabs, Ipswich, MA, USA) was used, with a reaction volume of $25 \mu \mathrm{L}$ comprising $1.5 \mu \mathrm{L}$ cDNA template and $0.5 \mu \mathrm{L}(10 \mu \mathrm{M})$ each of forward and reverse primers.

\section{DNA sequencing}

The PCR products were sequenced to detect the gene mutation in HER 2 and BRAF. Sequencing was performed in forward and reverse direction using the PCR products. Sequencing was conducted by BGI Tech Solutions Co., Ltd. (Shenzhen, China). Sequencing results were analyzed using the Basic Local Alignment Search Tool online database (https://blast.ncbi.nlm.nih.gov/Blast.cgi: accessed in May 2015) and sequencing data analysis software. BioEdit (version 7.0.9; Abbott Pharmaceutical Co., Lake Bluff, IL, UAS) and SeqMan NGen (version 11.2.1; DNASTAR, Madison, WI, USA) were used as sequence analyzing tools in the present study.

\section{Statistical analysis}

Numerical data were expressed as the mean \pm standard deviation. Chi-square test and Fisher's exact test were used to evaluate the statistical significance of the mutations with biological parameters using SPSS 17.0 (SPSS, Chicago, IL, USA). The $\mathrm{P}$ values were calculated based on two-sided hypothesis. $\mathrm{P}<0.05$ was considered to indicate a statistically significant difference.

\section{Results}

\section{Expression and gene mutation of HER2 in air pollution- related lung cancer}

Of the 82 tissue samples, 17 cancer samples exhibited the HER2 overexpression (Table 2). In the present study, the target gene was amplified in tumor specimens but not in the corresponding non-malignant counterparts. PCR amplification yielded clear bright bands with a fragment size of $\sim 1,100 \mathrm{bp}$ (Figure $1 A$ ). DNA sequencing analysis confirmed the specificity of amplicon; the result also revealed one positive case exhibited the HER2 mutation (insertion); the tissue sample of a female non-smoker aged 48 years and with a well-differentiated adenocarcinoma exhibited a novel 2866-2867 GGGCTCCCCG insertion in exon 20 (Figure 1B). This female patient resides in the clean region C. Additionally, HER2 overexpression was not statistically associated with air pollution levels, smoking status or total exposure degrees (Figure 2). Of the 13 cell lines, five lung cancer cell lines (A549, 95D, NCI-H446, NCI-H460 and SPC-A1) demonstrated HER 2 upregulation, but the target gene failed to amplify from the cDNA of 
Table 2 Association of HER2 overexpression, as well as EML4-ALK and CD74-ROS1 fusion with clinical features

\begin{tabular}{|c|c|c|c|c|}
\hline Features & Total No. & HER2 & $E M L 4-A L K$ & CD74-ROS1 \\
\hline Total subject & 82 & 17 & 5 & 3 \\
\hline \multicolumn{5}{|l|}{ Gender } \\
\hline Male & 49 & 9 & 4 & 0 \\
\hline Female & 33 & 8 & 1 & 3 \\
\hline \multicolumn{5}{|l|}{ Age (years) } \\
\hline$\leq 60$ & 63 & 13 & 4 & 2 \\
\hline$>60$ & 19 & 4 & 1 & 1 \\
\hline \multicolumn{5}{|l|}{ Air pollution degree } \\
\hline A: $\left(171-3,200 \mathrm{ng} / \mathrm{m}^{3}\right)$ & 34 & 7 & 2 & 1 \\
\hline B: $\left(60-170 \mathrm{ng} / \mathrm{m}^{3}\right)$ & 23 & 3 & 1 & 0 \\
\hline $\mathrm{C}:\left(16-60 \mathrm{ng} / \mathrm{m}^{3}\right)$ & 25 & 7 & 2 & 2 \\
\hline \multicolumn{5}{|l|}{ Smoking status } \\
\hline Ever & 37 & 9 & 4 & 0 \\
\hline Never & 45 & 8 & 1 & 3 \\
\hline \multicolumn{5}{|l|}{ Histology } \\
\hline$A C$ & 51 & 11 & 2 & 2 \\
\hline SCC & 31 & 6 & 3 & 1 \\
\hline
\end{tabular}

normal bronchial epithelial cell lines (Table 3).

\section{BRAF mutation in air pollution-related lung cancer}

All the 82 tissue samples and 13 cell lines were sequenced to identify BRAF mutation. However, no mutation was identified in any tissue sample and cell lines.

\section{EML4-ALK fusion in air pollution-related lung cancer}

There are 15 different $E M L 4-A L K$ fusion transcript variants identified to data (30). In the present study, all the tissue samples and the cell lines were screened to determine different variants of $E M L 4-A L K$ gene fusion. Overall, $6.1 \%$ $(5 / 82)$ of the tumor samples were positive for the EML4$A L K$ fusion and only two variants were detected (Table 2). A total of two cases were positive for EML4-E2+ALK-E20. The electrophoresis results revealed a clear and specific amplicon of $550 \mathrm{bp}$; therefore, the product was variant $5 \mathrm{~b}$. A total of three cases $(3.66 \%)$ were also positive for EML4-E13 + ALK-E20. The amplification product of 1,100 bp confirmed that these cases are variant 2 (Figure $3 A, B$ ). This fusion gene was more commonly identified in young male smokers (Table 2). The EML4-ALK fusion gene was not detected in the normal lung tissues adjacent to cancer tissues. Although the EML4-ALK fusion gene was not statistically associated with air pollution levels and smoking status $(\mathrm{P}=0.1)$, all the five cases occurred either in smokers or in patients from the most air-polluted region $\mathrm{A}$, and no positive cases were detected in non-smokers or patients from the relatively clean regions (Figure 3). The only cell line identified as positive for EML4-E2 + ALK-E20 was the LCC cell line (NCI-H446; Table 3).

\section{CD74-ROS1 fusion gene in air pollution-related lung cancer}

CD74-ROS1 gene fusion events were detected in 3.6\% (3/82) of the tumor samples; by contrast, CD74-ROS1 was not detected in the normal tissues adjacent to the cancer tissues. The amplicon size $630 \mathrm{bp}$ indicated the fusion of CD74-E6 + ROS1-E34 (Figure 3C,D). Notably, all three CD74-ROS1-positive patients were female non-smokers; two of them diagnosed as lung adenocarcinoma and one as SCC (Table 2). However, no statistical significance was observed between smokers and non-smokers, or between low total exposure and high total exposure groups (Figure 2). No CD74-ROS1-positive cell line was detected (Table 3).

\section{Discussion}

The incidence of air pollution-related lung cancer has increased over the previous three decades, particularly in China (11,31). Air pollution-related lung cancer has been considered as a deteriorating public health problem in China, particularly in highly polluted regions including Xuanwei and Fuyuan. These regions yield high indoor and outdoor air pollution levels because of smoky coal burning; furthermore, the prevalence of lung cancer in these regions is associated with air pollution. Moreover, the incidence of lung cancer among non-smoking female in Xuanwei is one of the highest rates worldwide (11-13). Thus, lung cancer in Xuanwei and Fuyuan populations provides a very good model to investigate air pollutionrelated lung cancer. Elucidating the molecular mechanisms of lung cancer occurrence in Xuanwei population would provide great significance for the clinical treatment of lung cancer. In addition, the majority of patients with lung $\mathrm{AD}$ who are East Asian never-smokers may potentially benefit 
A

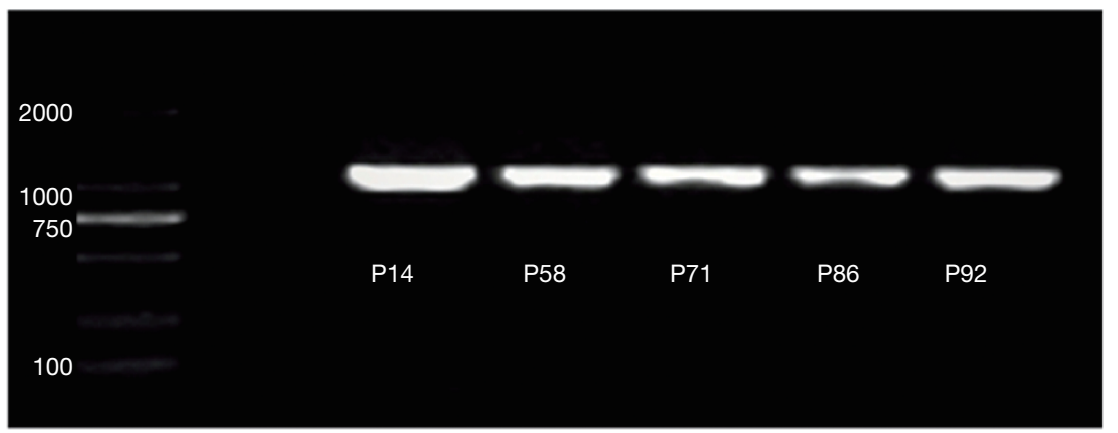

B

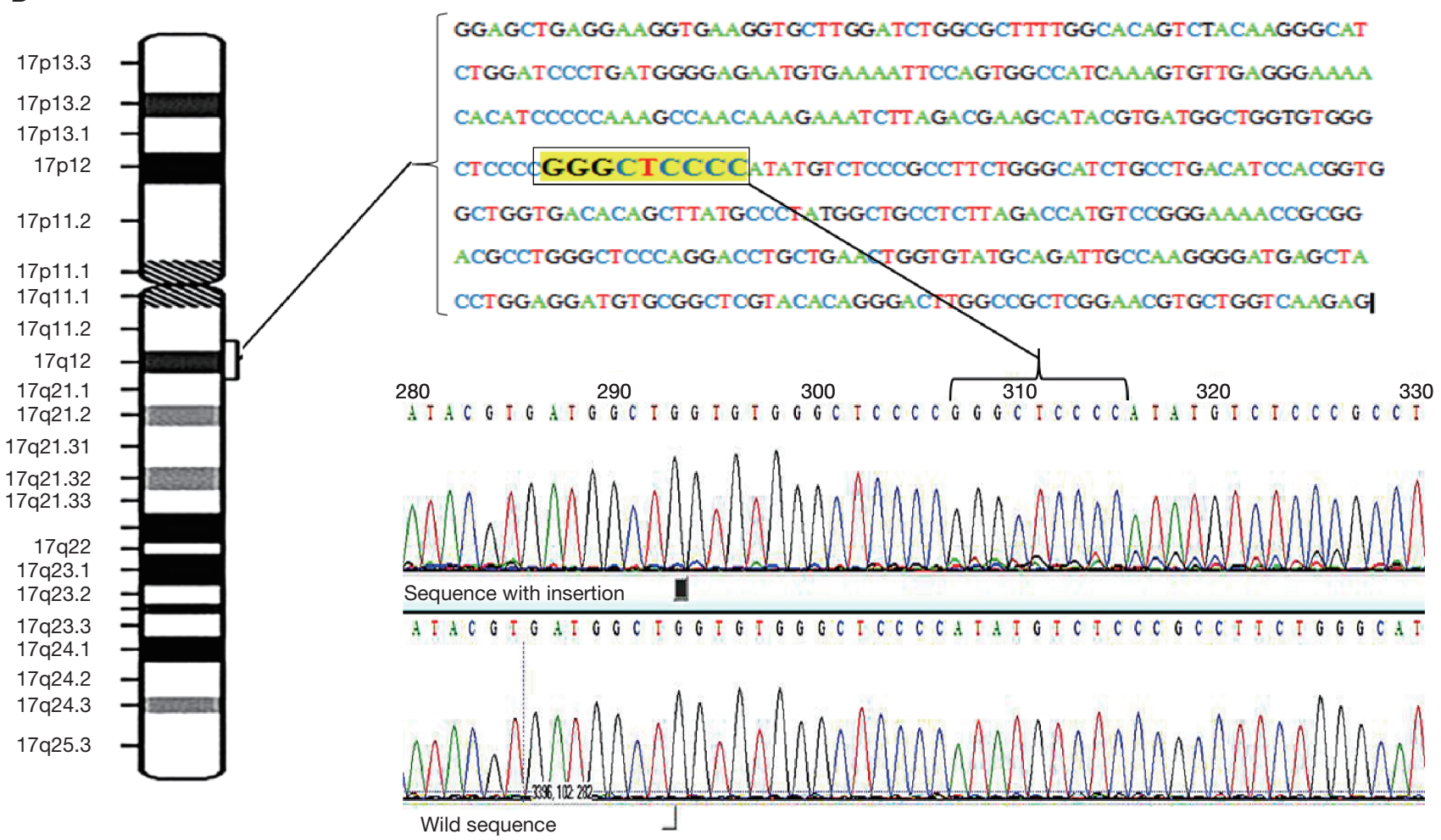

Figure 1 RT-PCR and DNA sequencing analyses of HER2 expression and mutations. (A) Electrophoresis results of HER2 overexpression in tumor samples using RT-PCR; (B) sequencing result of the novel mutation detected in a female patient with lung adenocarcinoma. P14, P58, P71, P86, and P92 represent sample numbers. RT-PCR, reverse transcription polymerase chain reaction; HER2, human epidermal growth factor receptor.

from targeted therapy (32). Therefore, clinically significant genetic changes in HER2, BRAF, EML4-ALK, and CD74ROS1 in the lung cancer cases in Xuanwei and Fuyuan were examined through a combination of RT-PCR and DNA sequencing in the present study.

HER2, also known as ErbB2 receptor tyrosine kinase 2, is a member of the ErbB family of receptor tyrosine kinases. $H E R 2$ has a critical role in cellular growth, proliferation, differentiation, apoptosis, and survival (33). In previous studies, HER 2 mutations were observed in $0-4 \%$ of NSCLCs; these mutations primarily involved insertions in exon 20 (25,34-36). Additionally, the HER2 kinase domain mutation rates are more frequent in female nonor light-smokers with lung $\mathrm{AD}$ compared with in other individuals $(34,35)$. HER2 mutations are associated with the overexpression of the HER2 protein and are considered as driver mutations in lung carcinogenesis (34). In the present study, a female non-smoker with lung $\mathrm{AD}$ in region $\mathrm{C}$ was 
A

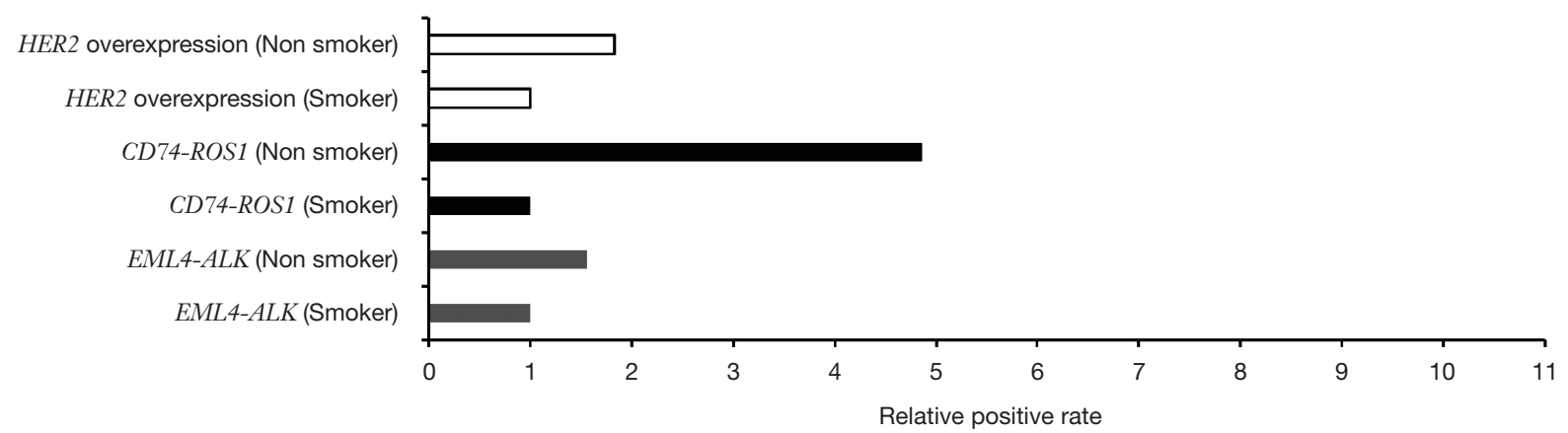

B

HER2 overexpression (Non smoker)
HER2 overexpression (Smoker)
CD74-ROS1 (Non smoker)
$C D 74-R O S 1$ (Smoker)
$E M L 4-A L K$ (Non smoker)
$E M L 4-A L K$ (Smoker)

C

HER2 overexpression (Low total exposure)

HER2 overexpression (High total exposure)

CD74-ROS1 (Low total exposure)

CD74-ROS1 (High total exposure)

EML4-ALK (Low total exposure)

EML4-ALK (High total exposure)

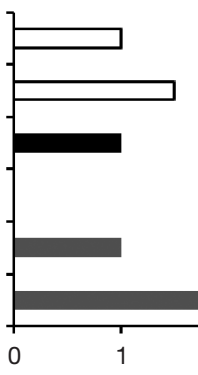

2

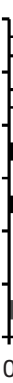

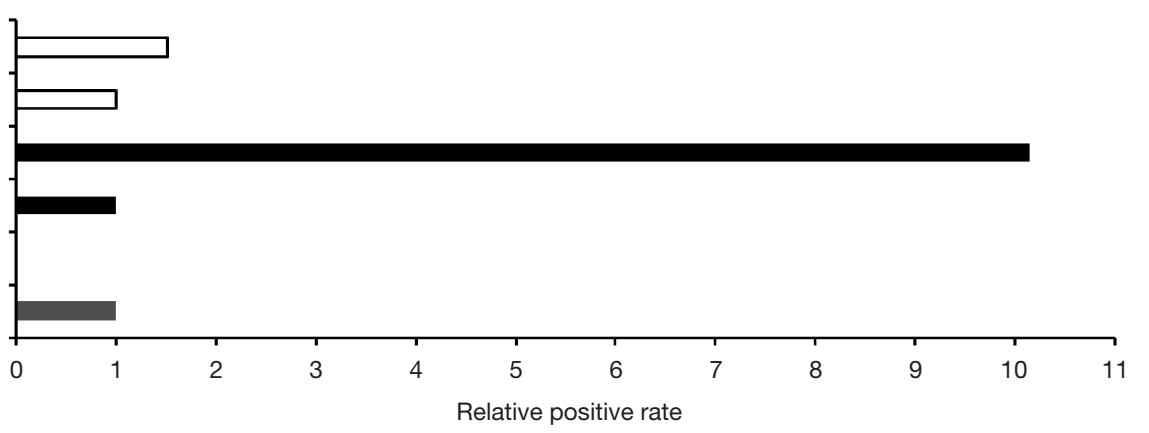

Figure 2 Association of HER2 overexpression, and EML4-ALK and CD74-ROS1 fusion genes with air pollution (A), smoking (B), and total exposure (C). Low air pollution, living in relatively clean region $\mathrm{C}$ (Bap concentration, $<60 \mathrm{ng} / \mathrm{m}^{3}$ ); High air pollution, living in heavily polluted regions A (Bap concentration, $>170 \mathrm{ng} / \mathrm{m}^{3}$ )/B (Bap concentration, $60-170 \mathrm{ng} / \mathrm{m}^{3}$ ). Low total exposure, living in relatively clean region $\mathrm{C}$ and also non-smoking; high total exposure, living in heavily polluted regions $\mathrm{A} / \mathrm{B}$ or smoking. HER2, human epidermal growth factor receptor; $E M L 4-A L K$, echinoderm microtubule-associated protein-like 4-anaplastic lymphoma kinase; CD74-ROS1, cluster of differentiation 74-ROS proto-oncogene 1; Bap, benzo(a)pyrene.

identified with a 9 bp novel insertion GGGCTCCCCG at 2866-2867 in exon 20. The adjacent non-malignant lung tissue presented a wild-type sequence of the HER2 gene; this result confirmed that this mutation was somatic. The complete HER 2 data from the present study indicated that the HER2 mutation may be one of the driver mutations in lung cancer and may serve an active role in the development of lung cancer. In Xuanwei, the concentration of Bap (the optimal index of PAH carcinogenicity) is higher than the national criterion for China due to air pollution (14). PAHs can directly bind to DNA, form DNA adducts, and induce gene mutations. A previous study confirmed the existence of PAH-DNA adducts in female patients with lung cancer in Xuanwei (11,37). In our previous study, 167 genes exhibited significantly higher mutation frequencies in patients from Xuanwei and Fuyuan compared with patients from control regions, and mutation rates of 70 genes were associated with the $\mathrm{BaP}$ exposure of patients (16); MUC16 mutations potentially associated with air pollution may participate in the development and progression of air pollution-related 
Table 3 Oncogenic alterations of cell lines included in the study

\begin{tabular}{lccc}
\hline Cell line & EML4-ALK & CD74-ROS1 & HER2 overexpression \\
\hline 801D & - & - & - \\
SPC-A-1 & - & - & + \\
GLC-82 & - & - & - \\
A549 & - & - & + \\
NCl-H292 & - & - & - \\
XLA-07 & - & - & - \\
XLJT & - & - & - \\
NCI-H460 & - & - & + \\
$95-D$ & - & - & + \\
NCI-H446 & + & - & + \\
16HBE & - & - & - \\
Beas-2B & - & - & - \\
\hline
\end{tabular}

+, positive; -, negative.

lung cancer (28). However, in the current study, it appeared that the HER2 mutation was not associated with $\mathrm{BaP}$ exposure. Furthermore, it was identified using RT-PCR that 17 cases of the lung cancer over-expressed HER2; by contrast, one of the 17 cases exhibited a HER 2 mutation. This finding suggested that numerous genetic mechanisms, including gene overrepresentation, translational and post-translational mechanisms, and gene mutation, may be responsible for HER 2 overexpression. In the present study, we also observed that HER2 overexpression was not associated with air pollution levels, smoking status, or total exposure degrees. Interestingly, the germline point mutation of HER2 (G660D) has been detected in non-smoker relatives with multigenerational lung AD in Japan (38). However, the germline mutation of HER2 was not found in familial lung cancer samples obtained from the Chinese population in highly air-polluted regions, Xuanwei and Fuyuan (39). Thus, we hypothesized that the HER2 mutation and HER2 overexpression may not be associated with BaP exposure. Nevertheless, the mechanisms underlying the HER2 mutation and overexpression in lung cancer require further investigated.

HER2 functions not only as a driver oncogene but also as a good target of personalized treatments. Patients with overexpressed HER 2 tumors exhibit sensitivity to Herceptin, which is a monoclonal antibody targeting HER2 (25).
HER2-targeted therapy has been successfully administered to treat breast, lung, and gastric cancer; and successful antitumor treatment with Herceptin is markedly associated with the HER2 overexpression in tumors $(25,40,41)$. Therefore, the examination of the HER2 expression level is a valuable test to screen patients for HER2-targeted therapy. Although FISH and IHC are commonly used in hospitals and laboratories for this particular examination, these methods have limitations at different levels (26). In the present study, RT-PCR was performed to evaluate the HER2 expression of the patients with lung cancer from Xuanwei and Fuyuan. The RT-PCR results demonstrated that 20.7\% (17/82) of the patients with lung cancer exhibited an upregulated HER 2 expression. In addition, the HER2 overexpression was more prevalent in females, smokers, and patients with lung $\mathrm{AD}$ compared with other patients. The results of the present study are consistent with other published observations through IHC and quantitative RT-PCR (qRTPCR); in particular, previous studies revealed that the HER2 overexpression was identified in $20 \%$ (29), 34.9\% (42) and $61.1 \%$ (43) of NSCLC using IHC, qRT-PCR and NGS (next generation sequencing), respectively. On the basis of these data, we hypothesized that: (I) certain lung cancer cases in Xuanwei and Fuyuan overexpressed HER2 in the same manner as the other lung cancer types; and (II) the HER2 expression level of the patients in Xuanwei and Fuyuan can be screened through RT-PCR-based methods. Consequently, the patients with HER2 overexpression may be effectively treated with appropriate therapies or clinical trials via effective screening, and therefore, patient survival may be improved. Although the association between the HER 2 overexpression and the air pollution was not observed, in view of its high prevalence $(20 \%)$ in air pollution-related lung cancer, it is worthwhile to screen patients for HER2 overexpression in this fatal disease for treatment guidelines. HER2 screening will be performed in cooperation with clinicians in hospitals in areas with high air pollution and high incidence of lung cancer in China. The mRNA overexpression of HER2 has been associated with prognosis of NSCLC $(18,42)$. Levels of HER2 mRNA may also be evaluated through qRT-PCR to determine the prognosis of patients with lung cancer.

$H E R 2$ and $B R A F$ encode proteins in the EGFR-signaling pathway; members of this pathway account for $>50 \%$ of somatic mutations in lung AD (44). Nevertheless, the estimated frequency of $B R A F$ mutations is quite low in lung cancer. Several studies have demonstrated that the 
A

Chromosome 2

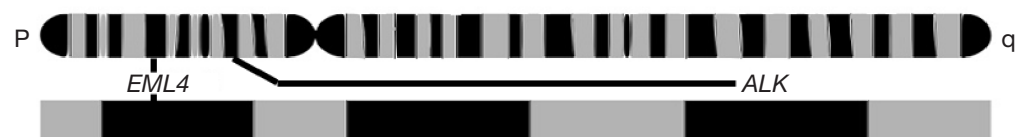

[IIIIIIIIIIIII

Exon 2

Exon 13

[IIIIIIIIIIIIIIII

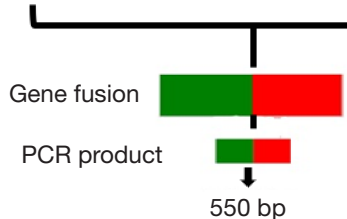

$+$

Exon 20
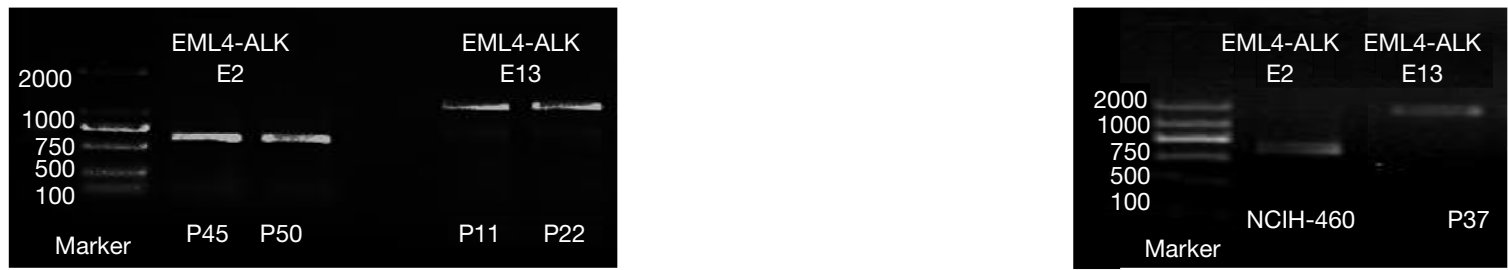

Gene fusion

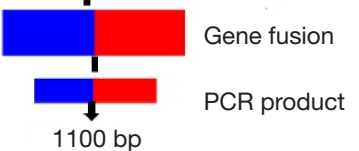

B

\footnotetext{
(1)
}

C

Chromosome 5

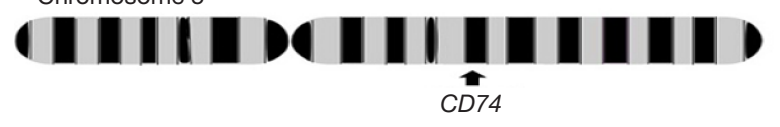

Q1 a a
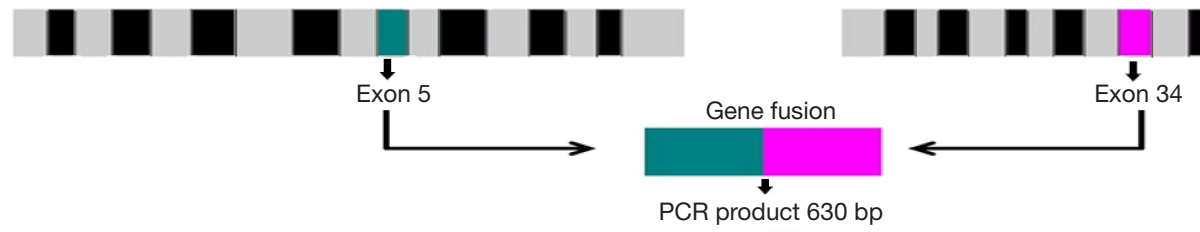

D

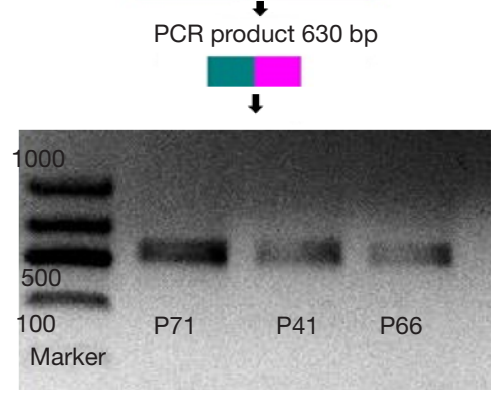

Figure 3 RT-PCR analysis of EML4-ALK and CD74-ROS1 fusion genes. (A) Graphical representation of EML4- $A L K$ fusion gene; (B) electrophoresis results of $E M L 4-A L K$ fusion gene in tumor tissue samples and cell line. Fragment size of 550 bp represent variant $5 \mathrm{~b}$ in EML4-E2+ALK-E20, whereas fragment size of 1,100 bp represent variant 2 in EML4-E13+ALK-E20; (C) graphical representation of CD74ROS1 fusion gene; (D) electrophoresis results of CD74-ROS1 fusion gene in tumor samples. RT-PCR, reverse transcription polymerase chain reaction; EML4-ALK, echinoderm microtubule-associated protein-like 4-anaplastic lymphoma kinase; CD74-ROS1, cluster of differentiation 74-ROS proto-oncogene 1. E, exon. P45, P50, P11, P22, P37, P71, P41, and P66 represent sample numbers.

common $B R A F$ mutation prevalence rate in populations with NSCLC is $1-4 \%(44,45)$, whereas a study in Caucasian patients revealed a higher prevalence rate of $\sim 9 \%$ (46). In addition, $B R A F$ mutations were predominantly found in current or past smokers with lung $\mathrm{AD}$ (45). In the present study, the $B R A F$ mutation was not detected in patients with lung cancer, similar to other published data that identified no mutation in the $B R A F$ gene of Asian non-smokers with lung cancer $(20,32)$. $B R A F$ mutations are potentially more common in lung cancer caused by smoking and are less 
prevalent in air pollution-related lung cancer.

The EML4-ALK fusion gene has been identified in lung cancer and several variants have been characterized to date $(30,47)$. This fusion gene potentially participates in lung carcinogenesis (47). In the present study, two variants of the EML4-ALK fusion gene were exhibited in $6 \%$ of patients with lung cancer in Xuanwei and Fuyuan. Furthermore, the present study also revealed that the patients harboring the EML4-ALK fusion gene were considerably younger compared with those without the fusion gene, which is similar to data from previous studies $(48,49)$. An additional study conducted in China identified that $5.8 \%(3 / 52)$ of lung cancer samples from non-smokers harbored this fusion gene (32). However, in the present study, EML4$A L K$ was detected to be more prevalent in smokers (10.8\%) compared with non-smokers (2.2\%). Notably, all the five cases occurred either in smokers or in patients from the most polluted region A; their respiratory system were considered to have suffered the highest levels of exposure to air carcinogens. Although the association between the exposure to air carcinogens and EML4-ALK fusion did not reached statistical significance, the effect of air carcinogens on the gene fusion should be considered.

Interestingly, previous studies identified ALK rearrangements primarily in lung $\mathrm{AD}(32,46)$; by contrast, the present study identified that the three cases of SCC also harbored the EML4-ALK fusion gene. The EML4$A L K$ fusion protein is among the most recent molecular targets of NSCLC therapy. Crizotinib, a small-molecule inhibitor designed for EML4-ALK fusion protein, yields an effective therapeutic outcome for patients with EML4$A L K(30)$. In the present study, 5 out of the 82 patients were positive for $E M L 4-A L K$; therefore, these patients may be treated with crizotinib. The present study also emphasized that the PCR-based test could detect not only the existence of fusion genes but also their different variants, which may affect responses to targeted inhibitors (48-50). Thus, the screening of the EML4-ALK fusion gene in air pollutionrelated lung cancer through RT-PCR may guide treatment.

CD74-ROS1 gene rearrangement is a novel oncogenic driver mutation. In the present study, the prevalence of this fusion in patients with lung cancer patients in Xuanwei and Fuyuan is $3.6 \%(3 / 82)$. The results of the present study indicated that CD74-ROS1-positive cases are more prevalent in non-smokers (6.6\%) and females (9.1\%). Similarly, ROS1 rearrangements in NSCLCs have been demonstrated to be more prevalent in young non-smokers as compared with smokers with lung $\mathrm{AD}$ (51). Furthermore, two out of three samples were positive for CD74-ROS1 fusion gene in the low total exposure group. Although the difference was not statistically significant due to the small number of positive cases, the observation indicated that air carcinogens may serve a minor role in the gene fusion event. The present study is only a pilot study, and further studies are required to achieve final conclusions.

Environmental pollution may induce its signature spectrum of oncogene mutations. However, air is fluid and it is able to affect a large area; therefore, air pollutionassociated mutations may be etiologically and clinically different from patterns of mutations induced by other environmental sources of carcinogens, including food, habits or occupational exposure, which may have more individual effects that are limited to certain types of mutation $(16,20)$. In addition, air pollution comprises a complicated mixture of chemicals that waft from sources ranging from factories to lawnmowers, and the components of air pollution differ in various regions $(5,10)$. Therefore, the spectrum of oncogene mutations caused by air pollution is extremely complex and requires extensive studies to demonstrate associations. Finally, air pollution and smoking collectively effect population, for examples: a smoker living in a clean region is exposed to carcinogens of a higher concentration due to smoking as compared with the non-smoker from a clean region, while a non-smoker living in a heavily polluted area is inevitably affected by higher level of air carcinogens as compared with the non-smoker from a clean region. Therefore, smoking and air pollution should be jointly considered in researches.

\section{Conclusions}

Several oncogenic alterations, including HER2 overexpression and mutation, and EML4-ALK and CD74-ROS1 fusion genes, were detected in air pollution-related lung cancer in Xuanwei and Fuyuan. These genetic alterations may be associated with air pollution-induced lung carcinogenesis; conversely, they may serve as molecular targets for personalized therapies. Therefore, the present study proposed that Herceptin and crizotinib could also be used in patients with air-pollution related lung cancer with HER2 overexpression and EML4-ALK fusion genes, respectively; and that RT-PCR-based test may be a useful tool to screen these genetic alterations in clinical application.

\section{Acknowledgments}

The authors gratefully acknowledge Dr. Hongbin Ji 
(Shanghai Institute of Biological Sciences, Chinese Academy of Sciences, Beijing) for providing the idea to write the current article. We thank Dr. Ping Wang (Department of Surgery, Yunnan First People's Hospital) and Dr. Yun-Chao Huang (Department of Thoracic and Cardiovascular Surgery, Yunnan Tumor Hospital) for providing the surgical samples. Funding: This study was supported by the Natural Science Foundation of China (grant number 81272617), the 973 Program (grant number 2011CB510104), the Special Fund for Local Science and Technology Development Guided by the Chinese Government (grant number 2018L3011) and sponsored by Chinese Academy of Sciences-the World Academy of Sciences President's Fellowship for International PhD Students (MK).

\section{Footnote}

Conflicts of Interest: All authors have completed the ICMJE uniform disclosure form (available at http://dx.doi. org/10.21037/tcr-19-1314). The authors have no conflicts of interest to declare.

Ethical Statement: The authors are accountable for all aspects of the work in ensuring that questions related to the accuracy or integrity of any part of the work are appropriately investigated and resolved. This study was approved by the Ethics Committee for Human Medicine Research at the Kunming Institute of Zoology, Chinese Academy of Sciences (Permit Number: SYDW-2012010). All patient records and information were anonymized and de-identified prior to analysis. Research was conducted in accordance with the principles of the Declaration of Helsinki (as revised in 2013). Informed consent was taken from all individual participants.

Open Access Statement: This is an Open Access article distributed in accordance with the Creative Commons Attribution-NonCommercial-NoDerivs 4.0 International License (CC BY-NC-ND 4.0), which permits the noncommercial replication and distribution of the article with the strict proviso that no changes or edits are made and the original work is properly cited (including links to both the formal publication through the relevant DOI and the license). See: https://creativecommons.org/licenses/by-nc-nd/4.0/.

\section{References}

1. Torre LA, Bray F, Siegel RL, et al. Global cancer statistics,
2012. CA Cancer J Clin 2015;65:87-108.

2. Wong MCS, Lao XQ, Ho KF, et al. Incidence and mortality of lung cancer: global trends and association with socioeconomic status. Sci Rep 2017;7:14300.

3. Brambilla E, Travis WD, Colby TV, et al. The new World Health Organization classification of lung tumours. Eur Respir J 2001;18:1059-68.

4. Pallis AG, Syrigos KN. Lung cancer in never-smokers: Disease characteristics and risk factors. Crit Rev Oncol Hematol 2013;88:494-503.

5. Deweerdt S. Aetiology: Crucial clues. Nature 2014;513:S12-13.

6. IHME. Global Burden of Disease 2010 (GBD 2010). Population Estimates 1970-2010. Institute for Health Metrics and Evaluation, Seattle, WA 2012.

7. Loomis D, Grosse Y, Lauby-Secretan B, et al. The carcinogenicity of outdoor air pollution. Lancet Oncol 2013;14:1262-3.

8. Straif KCA, Samet J. Air Pollution and Cancer. IARC Scientific Publication No. 161. Lyon: IARC Press, 2013.

9. Chen Z, Wang JN, Ma GX, et al. China tackles the health effects of air pollution. Lancet 2013;382:1959-60.

10. Subbaraman N. Public health: A burning issue. Nature 2014;513:S16-17.

11. Wong JYY, Downward GS, Hu W, et al. Lung cancer risk by geologic coal deposits: A case-control study of female never-smokers from Xuanwei and Fuyuan, China. Int J Cancer 2019;144:2918-27.

12. Cao Y, Gao H. Prevalence and causes of air pollution and lung cancer in Xuanwei City and Fuyuan County, Yunnan Province, China. Front Med 2012;6:217-20.

13. Mumford JL, He XZ, Chapman RS, et al. Lung cancer and indoor air pollution in Xuan Wei, China. Science 1987;235:217-20.

14. Lv J, Xu R, Wu G, et al. Indoor and outdoor air pollution of polycyclic aromatic hydrocarbons (PAHs) in Xuanwei and Fuyuan, China. J Environ Monit 2009;11:1368-74.

15. Li J, Zhu X, Yu K, et al. Exposure to Polycyclic Aromatic Hydrocarbons and Accelerated DNA Methylation Aging. Environ Health Perspect 2018;126:067005.

16. Yu XJ, Yang MJ, Zhou B, et al. Characterization of Somatic Mutations in Air Pollution-Related Lung Cancer. EBioMedicine 2015;2:583-90.

17. Pao W, Kris MG, Iafrate AJ, et al. Integration of molecular profiling into the lung cancer clinic. Clin Cancer Res 2009;15:5317-22.

18. Gatzemeier U, Groth G, Butts C, et al. Randomized phase II trial of gemcitabine-cisplatin with or without 
trastuzumab in HER2-positive non-small-cell lung cancer. Ann Oncol 2004;15:19-27.

19. Shaw AT, Yeap BY, Solomon BJ, et al. Effect of crizotinib on overall survival in patients with advanced non-smallcell lung cancer harbouring ALK gene rearrangement: a retrospective analysis. Lancet Oncol 2011;12:1004-12.

20. Hosgood HD 3rd, Pao W, Rothman N, et al. Driver mutations among never smoking female lung cancer tissues in China identify unique EGFR and KRAS mutation pattern associated with household coal burning. Respir Med 2013;107:1755-62.

21. Keohavong P, Gao WM, Zheng KC, et al. Detection of $\mathrm{K}$-ras and p53 mutations in sputum samples of lung cancer patients using laser capture microdissection microscope and mutation analysis. Anal Biochem 2004;324:92-9.

22. Wu YC, Chang IC, Wang CL, et al. Comparison of IHC, FISH and RT-PCR methods for detection of ALK rearrangements in 312 non-small cell lung cancer patients in Taiwan. PLoS One 2013;8:e70839.

23. Wang J, Cai Y, Dong Y, et al. Clinical characteristics and outcomes of patients with primary lung adenocarcinoma harboring ALK rearrangements detected by FISH, IHC, and RT-PCR. PLoS One 2014;9:e101551.

24. Heredia NJ, Belgrader P, Wang S, et al. Droplet Digital PCR quantitation of HER2 expression in FFPE breast cancer samples. Methods 2013;59:S20-23.

25. Denkert C, Huober J, Loibl S, et al. HER2 and ESR1 mRNA expression levels and response to neoadjuvant trastuzumab plus chemotherapy in patients with primary breast cancer. Breast Cancer Res 2013;15:R11.

26. Hillig T, Thode J, Breinholt MF, et al. Assessing HER2 amplification by IHC, FISH, and real-time polymerase chain reaction analysis (real-time PCR) following LCM in formalin-fixed paraffin embedded tissue from 40 women with ovarian cancer. APMIS 2012;120:1000-7.

27. Jiang CL, He SW, Zhang YD, et al. Air pollution and DNA methylation alterations in lung cancer: A systematic and comparative study. Oncotarget 2017;8:1369-91.

28. Kanwal M, Ding X, Song X, et al. MUC16 overexpression induced by gene mutations promotes the growth and invasion of lung cancer cells. Oncotarget 2018;9:12226-39.

29. Heinmöller P, Gross C, Beyser K, et al. HER2 status in non-small cell lung cancer: results from patient screening for enrollment to a phase II study of herceptin. Clin Cancer Res 2003;9:5238-43.

30. Maus MK, Stephens C, Zeger G, et al. Identification of novel variant of EML4-ALK fusion gene in NSCLC: Potential benefits of the RT-PCR method. Int J Biomed
Sci 2012;8:1-6.

31. Huang YB, Song FJ, Liu Q, et al. A bird's eye view of the air pollution-cancer link in China. Chin J Cancer 2014;33:176-88.

32. Sun $Y$, Ren $Y$, Fang Z, et al. Lung adenocarcinoma from East Asian never-smokers is a disease largely defined by targetable oncogenic mutant kinases. J Clin Oncol 2010;28:4616-20.

33. Wieduwilt MJ, Moasser MM. The epidermal growth factor receptor family: biology driving targeted therapeutics. Cell Mol Life Sci 2008;65:1566-84.

34. Sasaki H, Shimizu S, Endo K, et al. EGFR and erbB2 mutation status in Japanese lung cancer patients. Int J Cancer 2006;118:180-4.

35. Shimamura T, Ji H, Minami Y, et al. Non-small-cell lung cancer and $\mathrm{Ba} / \mathrm{F} 3$ transformed cells harboring the ERBB2 G776insV_G/C mutation are sensitive to the dual-specific epidermal growth factor receptor and ERBB2 inhibitor HKI-272. Cancer Res 2006;66:6487-91.

36. Shi Y, Wang M. Afatinib as first-line treatment for advanced lung adenocarcinoma patients harboring HER2 mutation: A case report and review of the literature. Thorac Cancer 2018;9:1788-94.

37. Yang K, Huang Y, Zhao G, et al. Expression of PAH-DNA adducts in lung tissues of Xuanwei female lung cancer patients. Zhongguo Fei Ai Za Zhi 2010;13:517-21.

38. Yamamoto H, Higasa K, Sakaguchi M, et al. Novel germline mutation in the transmembrane domain of HER2 in familial lung adenocarcinomas. J Natl Cancer Inst 2014;106:djt338.

39. Kanwal M, Ding X, Ma ZH, et al. Characterization of germline mutations in familial lung cancer from the Chinese population. Gene 2018;641:94-104.

40. Mar N, Vredenburgh JJ, Wasser JS. Targeting HER2 in the treatment of non-small cell lung cancer. Lung Cancer 2015;87:220-5.

41. Ock CY, Lee KW, Kim JW, et al. Optimal Patient Selection for Trastuzumab Treatment in HER2Positive Advanced Gastric Cancer. Clin Cancer Res 2015;21:2520-9.

42. Brabender J, Danenberg KD, Metzger R, et al. Epidermal growth factor receptor and HER2-neu mRNA expression in non-small cell lung cancer Is correlated with survival. Clin Cancer Res 2001;7:1850-5.

43. Lee K, Jung HA, Sun JM, et al. Clinical Characteristics and Outcomes of Non-small Cell Lung Cancer Patients with HER2 Alterations in Korea. Cancer Res Treat 2020;52:292-300. 
44. Marks JL, McLellan MD, Zakowski MF, et al. Mutational analysis of EGFR and related signaling pathway genes in lung adenocarcinomas identifies a novel somatic kinase domain mutation in FGFR4. PLoS One 2007;2:e426.

45. Paik PK, Arcila ME, Fara M, et al. Clinical characteristics of patients with lung adenocarcinomas harboring BRAF mutations. J Clin Oncol 2011;29:2046-51.

46. Ilie $\mathrm{M}$, Long E, Hofman V, et al. Diagnostic value of immunohistochemistry for the detection of the BRAFV600E mutation in primary lung adenocarcinoma Caucasian patients. Ann Oncol 2013;24:742-8.

47. Takahashi T, Sonobe M, Kobayashi M, et al.

Clinicopathologic features of non-small-cell lung cancer with EML4-ALK fusion gene. Ann Surg Oncol

Cite this article as: Kanwal $M$, Ding $\mathrm{X}$, Lin W, Cao Y. Screening of significant oncogenic changes in air pollutionrelated lung cancer in Chinese population. Transl Cancer Res 2020;9(7):4341-4353. doi: 10.21037/tcr-19-1314
2010;17:889-97.

48. Teixidó C, Karachaliou N, Peg V, et al. Concordance of IHC, FISH and RT-PCR for EML4-ALK rearrangements. Transl Lung Cancer Res 2014;3:70-4.

49. Shaw AT, Yeap BY, Mino-Kenudson M, et al. Clinical features and outcome of patients with non-small-cell lung cancer who harbor EML4-ALK. J Clin Oncol 2009;27:4247-53.

50. Soda M, Choi YL, Enomoto M, et al. Identification of the transforming EML4-ALK fusion gene in non-small-cell lung cancer. Nature 2007;448:561-6.

51. Bergethon K, Shaw AT, Ou SH, et al. ROS1 rearrangements define a unique molecular class of lung cancers. J Clin Oncol 2012;30:863-70. 